\title{
Tecnologías y Educación: Su Percepción Social En Santiago de Chile
}

\author{
Arturo B. Rodriguez ${ }^{(1)}$, Leonardo J. Ramirez ${ }^{(2)}$ y Felipe R. M. Basile ${ }^{(3)}$ \\ (1) Universidad Santiago de Chile, Depto. de Tecnología Industrial, Grupo de Investigación en Nuevas \\ Tecnologías (GINT), Santiago de Chile, Chile (email: arturo.rodriguez@usach.cl) \\ (2) Universidad Militar de Nueva Granada, Grupo de Investigación en Telemedicina (TIGUM), \\ Bogotá, Colombia. (email: leonardo.ramirez@unimilitar.edu.co) \\ (3) Instituto Federal de Educação, Ciência e Tecnologia, São Paulo, Brasil \\ (e-mail: felipe.basile@ifsp.edu.br)
}

Recibido Abr. 28, 2017; Aceptado Jul. 6, 2017; Versión final Ago. 8, 2017, Publicado Dic. 2017

\begin{abstract}
Resumen
El artículo muestra los resultados de un estudio realizado en tres comunas de la Región MetropolitanaChile, donde se encuestaron en forma presencial a 1228 personas de diferentes grupos etarios, laborales, nivel educativo y de género. El instrumento validado se compone de cuatro dimensiones: estudiantes, docencia, calidad de la enseñanza y relaciones interpersonales y con la tecnología. El instrumento fue diseñado para conocer la percepción de la población sobre la relación existente entre Educación y Tecnología. El análisis mostró una sociedad que permite el uso tecnológico en los procesos educativos, coincidente con estudios previos publicados. Sin embargo, existe escepticismo sobre el aporte de la tecnología en la mejora de las relaciones interpersonales y la calidad de la enseñanza. Otro resultado interesante es que existe en la población una postura clara frente a la tecnología, sea a favor o en contra de ella. Solamente el $3 \%$ de la población es indiferente al tema abordado.
\end{abstract}

Palabras clave: tecnología; educación; calidad de la enseñanza; tecnología de la información y la comunicación.

\section{Technologies and Education: Their Social Perception in Santiago de Chile}

\begin{abstract}
The article shows the results of a study developed in three areas of the Metropolitan Region in Chile, in which 1228 people of different age, labor, educational level, and gender were surveyed. The validated instrument consists of four dimensions students, teaching, teaching quality, interpersonal relations and relationship with technology. The instrument was designed to know the perception of the population about the relationship between education and technology. The analysis showed a society that is permissive with respect of the introduction of technologies in the educational processes, in agreement with previous studies. However, there is skepticism about the contribution of technology for improving interpersonal relations. Another interesting result is that there is a clear interest and position of the people about technology, either in favor or against it. Only $3 \%$ of the population surveyed has an indifferent position.
\end{abstract}

Keywords: technology; education; quality of teaching; information technology and communication. 


\section{INTRODUCCIÓN}

Desde la aparición y desarrollo de la informática y las telecomunicaciones, todas las actividades humanas han soportado una incesante incorporación tecnológica, siendo la educación una de las actividades con mayor inserción tecnológica en la última década. La sociedad en su conjunto ha sido en general permeable a esta introducción sin embargo en los últimos años, diversas instituciones y grupos ciudadanos han mostrado cierta preocupación frente al uso de la tecnología, poniendo en evidencia la percepción (Rodríguez et al.; 2016). Por otro lado el uso de las TIC han sido y son preocupación actual de los investigadores, donde la utilización heterogénea de los recursos TIC van desde solo el uso de los email hasta el uso de complejos simuladores en la sala de clase (Lagunes-Dominguez et al., 2015).

La literatura muestra investigaciones sobre la diferencia de conocimientos en TIC que existe entre estudiantes y Docentes, siendo los estudiantes los que tienen más habilidades en esta tecnología valorando con mayor intensidad el uso de las herramientas informáticas, la sociedad del futuro demanda un nuevo tipo de ciudadano cuya formación es responsabilidad del sistema educativo actual (Cózar-Gutiérrez et al., 2016). También muchos autores han investigado la tecnología y su impacto en la educación superior y desde la perspectiva de la gestión se incorporaron los llamados LMS - Learning Management Systems o Sistemas de Gestión del Aprendizaje donde la fiabilidad de los LMS están sujetos a las condiciones de hardware, la capacidad de gestión docente y del estudiante (Moreno, 2017; Polivio et al., 2016). Estos sistemas establecen un aumento de la impersonalidad entregando la responsabilidad al sistema de gestión olvidando que las personas se relacionan con personas y no con sistemas ayudando a deformar la percepción de un Sistema educativo de calidad. Además, desde la perspectiva del proceso enseñanza-aprendizaje los defensores de las tecnologías en la educación manifiestan que la educación busca a través de las TIC sobrepasar sus orígenes, es decir establecer y garantizar un espacio de aprendizaje que promueva el diálogo crítico con una cultura más horizontal y menos estricta, que apueste por la investigación y una participación activa de los protagonistas del proceso y los recursos abiertos es una forma de lograrlo. (Starobinas, 2013).

Por ejemplo, los esfuerzos del Instituto Tecnológico de Monterrey al desarrollar el portal de recursos abiertos TEMOA (La palabra "TEMOA" significa "buscar, investigar, indagar" en la lengua Náhuatl), que permitió indexar una gran cantidad de recursos educativos para el libre acceso, un estudio profundo puede ser visto en (Mortera, 2010). Además con la integración de las TIC los docentes han desarrollado estrategias que pueden intercambiar a partir las redes nacionales e internacionales logrando modificar positivamente sus actividades de enseñanza, Comunicación, gestión, vinculación, e investigación (López de la Madrid, 2013). Otros autores explican la ventaja de las tecnologías basados en la demanda de ciertas habilidades tecnológica que el mercado requiere. La incorporación de las TIC en los centros educativos permite a estudiantes y docentes acceso a cualquier información, entonces aparece la enseñanza personalizada, centrada en el estudiante y basada en el socioconstructivismo pedagógico, que asegura a los estudiantes las competencias en TIC que la sociedad demanda y otras tan importantes como la curiosidad y el aprender a aprender, la iniciativa y responsabilidad, el trabajo en equipo (Márquez, 2013).

Los estudiantes actuales tienen una postura frente al uso de las tecnologías y las perciben como herramientas indispensables necesarias para estar al día y lograr adaptarse e insertase a la vida laboral y consideran que los docentes no han realizado los suficientes esfuerzos para incorporarlos en sus habilidades (Silva-Peña, 2006). Por otro lado, la educación a distancia ha evolucionado desde posiciones unívocas a posiciones multivócas y colaborativas donde el estudiante es protagonista activo de su formación, mientras más activo es el proceso el anclaje significativo es mayor (López, 2009). Sin embargo, los docentes tienen una resistencia al cambio que no permite que la tecnología llegue a la sala de clases, los docentes solo cambiarán si logran observar los beneficios de hacerlo y las desventajas de no hacerlo (Bates, 2001). Además el hablar de innovación educativa con base tecnológica no solo implica que exista el equipamiento de hardware asociado, sino también, las Tecnologías Digitales, Software y Ambientes de Aprendizaje para una modificación de los procesos académicos y administrativos y sin duda el compromiso y conducta de cambio del personal docente (López et al., 2013, Tapasco et al., 2017;Araneda-Guirriman et al., 2016; Rodriguez-Ponce et al., 2016).

Desde lo expuesto la tecnología está muy presente en la educación, sin embargo la población tiene una percepción, respecto del impacto que esta ha tenido y tiene en la educación. Por lo demás, los cambios que se realicen solo serán efectivos si se cambian los métodos y concepciones sobre la enseñanza, y el protagonismo horizontal de los actores del proceso educativo (Cabero, 2005). El trabajo muestra esta percepción relacionada con la tecnología como aporte a la educación y formación de sus hijos de una población cada vez más heterogénea y cambiante. 


\section{METODOLOGIA}

Se seleccionaron tres zonas de intervención, Puente Alto, Nuñoa y Las Condes, que tienen diferentes grupos socioeconómicos predominantes según datos INE de acuerdo a la tupla porcentual $(A B C 1, C 2, C 3$, D, E) con la comuna Puente Alto $(4,0 \% 18,9 \% 31,1 \% 38,1 \% 7,9 \%)$, Nuñoa $(28,9 \% 36,3 \% 19,0 \% 13,5 \%$ $2,4 \%$ ) y Las condes $(53,5 \% 30,0 \% 9,6 \% 6,1 \% 0,9 \%)$, se realizaron en total 1228 encuestas presenciales, 476 en la comuna de Puente Alto, 421 en la comuna de Ñuñoa y 331 en la Comuna de las Condes.

El instrumento interventor es una encuesta de 12 preguntas y una escala de cinco niveles Totalmente de Acuerdo (TDA), Relativamente de Acuerdo (RDA), Relativamente en Desacuerdo (RED), Totalmente en Desacuerdo (TED) e Indiferente (IND) validada por expertos del área psicológica y de especialista en estadística no paramétrica todos con más de 7 años de experiencia en su campo (Rodríguez et al., 2016). La intervención fue simultánea con un equipo estudiantes becados (encuestadores) previamente capacitados junto a tres supervisores de encuestas con una muestra de control aleatorio, una por cada comuna. Los estudiantes encuestadores son de diferentes departamentos y facultades para no generar sesgos, maximizar el control y mejorar la ejecución del proceso. La totalidad del proceso estuvo controlado y supervisado por el equipo investigador. La intervención se realizó en tres etapas debido al gran número de encuestas. Se establecieron cuatro dimensiones asociadas a las doce preguntas, Estudiantes y TIC (ESTIC) esta dimensión permite observar la percepción de la relación estudiantes-tecnología; Docencia y TIC (DOTIC), esta dimensión permite observar la percepción de la relación Docente-tecnología; Relaciones Interpersonales y TIC (RITIC), esta dimensión permite observar la percepción de las relaciones interpersonales y la tecnología y Calidad Educativa y TIC (CETIC), esta dimensión permite observar la percepción de la relación Calidad y tecnología.

La muestra intervenida es de 1228 entrevistas individuales y presenciales de un total poblacional de 1.114.314 individuos (Fuente: INE Instituto nacional de Estadística) asociadas a las comunas de Puente Alto (PA), Ñuñoa (ÑU) y Las Condes (LC). En la Tabla 1, se observa la distribución porcentual diferenciada por género, la similitud de la composición garantiza la comparación. El género femenino $(F)$ alcanza un $51 \%$ y el masculino (M) un $49 \%$ en promedio. En la Tabla 2, se presenta la distribución porcentual de las muestras respecto del nivel educativo para cada zona de estudio. Se utilizaron tres niveles, nivel básico, medio y profesional, la similitud de las muestras garantizan la correcta comparación.

Tabla 1: Distribución porcentual de la muestra por género y grupo etario en las tres comunas.

\begin{tabular}{|c|c|c|c|c|c|c|}
\hline \multirow{2}{*}{$N$} & \multicolumn{2}{|c|}{476} & \multicolumn{2}{c|}{421} & \multicolumn{2}{c|}{331} \\
\hline \multirow{2}{*}{ Años } & \multicolumn{2}{|c|}{ Puente Alto } & \multicolumn{2}{c|}{ Nuñoa } & \multicolumn{2}{c|}{ Las Condes } \\
\cline { 2 - 7 } & $\mathrm{M}$ & $\mathrm{F}$ & $\mathrm{M}$ & $\mathrm{F}$ & $\mathrm{M}$ & $\mathrm{F}$ \\
\hline $18-29$ & 11,6 & 11,8 & 12,4 & 10,5 & 14,5 & 13,9 \\
\hline $30-44$ & 16,8 & 18,1 & 14,7 & 12,4 & 13,0 & 13,0 \\
\hline $45-59$ & 11,3 & 11,8 & 14,3 & 9,3 & 13,6 & 13,0 \\
\hline $60-74$ & 6,5 & 7,4 & 10,5 & 11,6 & 7,9 & 9,1 \\
\hline 75 o + & 2,3 & 2,5 & 2,9 & 1,7 & 0,9 & 1,2 \\
\hline Subtotal & 48,5 & 51,5 & 54,6 & 45,4 & 49,8 & 50,2 \\
\hline
\end{tabular}

Tabla 2: Distribución porcentual de la muestra por nivel educativo en las tres comunas.

\begin{tabular}{|l|c|c|c|c|}
\hline \multicolumn{2}{|c|}{$N$} & 476 & 421 & 331 \\
\hline Nivel Educativo & ABR. & PA & $\tilde{N} U$ & LC \\
\hline Básico & BA & 4,62 & 0,71 & 0,60 \\
\hline Medio & ME & 37,82 & 31,12 & 21,15 \\
\hline Profesional & PR & 57,56 & 68,17 & 78,25 \\
\hline
\end{tabular}

La característica de la muestra poblacional utilizando cuatro variables tales como: Comuna, Género, Grupo Etario y Nivel Educacional con cuatro dimensiones tales como: ESTIC, DOTIC, RITIC Y CETIC, permite un marco de similitud que garantizan una comparación válida con resultados minimizando el sesgo. Por lo demás las mediciones fueron establecidas presencialmente y solo indican una percepción instantánea y de 
ninguna manera este estudio pretende aseverar o asegurar que es una conducta permanente, para establecer un resultado concluyente se deberá realizar un estudio de mayor extensión y envergadura.

Solo para mostrar la distribución económica de las zonas de estudio se registró el área laboral de la muestra y se determinaron ocho atributos para esta variable, tales como: Estudiante (ES), Casa (CA), Telecomunicaciones (TL), Construcción (CO), Industria (IN), Agricultura (AG), Educación (ED), y Otros (OT). En la Tabla 3, se distingue la distribución porcentual heterogénea del área laboral debido a que las zonas estudiadas corresponden a ingresos económicos diferenciados lo que se nota en la distribución profesional y educativa de los encuestados.

Tabla 3: Distribución porcentual de la muestra por área laboral en las tres comunas.

\begin{tabular}{|l|c|c|c|c|}
\hline \multicolumn{2}{|c|}{$\mathrm{N}$} & 476 & 421 & 331 \\
\hline Área Laboral & $A B R$. & $P A$ & $\tilde{N} U$ & $L C$ \\
\hline Estudiante & ES & 13,66 & 9,03 & 17,82 \\
\hline Casa & CA & 14,50 & 18,53 & 13,60 \\
\hline Telecomunicaciones & TL & 5,67 & 4,51 & 5,44 \\
\hline Construcción & CO & 17,23 & 14,49 & 12,08 \\
\hline Industria & IN & 9,24 & 13,06 & 13,90 \\
\hline Agricultura & AG & 2,94 & 2,61 & 2,42 \\
\hline Educación & ED & 12,18 & 9,98 & 9,37 \\
\hline Otros & OT & 24,58 & 27,79 & 25,38 \\
\hline
\end{tabular}

\section{ANALISIS Y RESULTADOS}

En la Figura 1, se observa que los jóvenes son los usuarios de mayor consumo de internet y disminuye a medida que el grupo etario es mayor. Por otro lado, la educación es la tercera razón para mantener el Internet en el hogar, siendo la comunicación la primera razón, esto transparenta la importancia que tiene la cultura y educación en la sociedad, donde es más importante la noticia cotidiana que el conocimiento desarrollado y que permite la solución de los problemas de la sociedad; es la representación de la individualidad de nuestra nueva sociedad (Pinto de la Fuente, 2016).

En la Figura 2, se puede observar la distribución similar de la percepción positiva de las dimensiones estudiadas en las comunas Puente Alto y Las Condes con una tendencia en alta de la dimensión ESTIC para el caso de Las Condes debido a que la coordenada ESTIC llega a valores cercanos al $80 \%$, sin embargo la comuna de Ñuñoa tiene una marcada diferencia en las dimensiones ESTIC y DOTIC que corresponde a la percepción positiva del impacto de la tecnología en los estudiantes y los docentes, sin embargo esta comuna no percibe de la misma forma la Calidad Educativa y las relaciones interpersonales. La misma tendencia se observa para la percepción negativa, nuevamente aparece la similitud en las mismas comunas, siendo la comuna de Ñuñoa que percibe un impacto negativo de las tecnologías en la Calidad Educativa y las relaciones interpersonales. Por otro lado, el área mostrada en la Comuna de Ñuñoa es un trapecio para la percepción positiva y negativa, mientras que en los otros casos es un trapezoide, esto indica la simetría de las respuestas de las dimensiones ESTIC y DOTIC para el caso de Ñuñoa. Por otro lado, las dimensiones RITIC y CETIC son percibidas como negativas en el caso de las tres zonas de intervención.

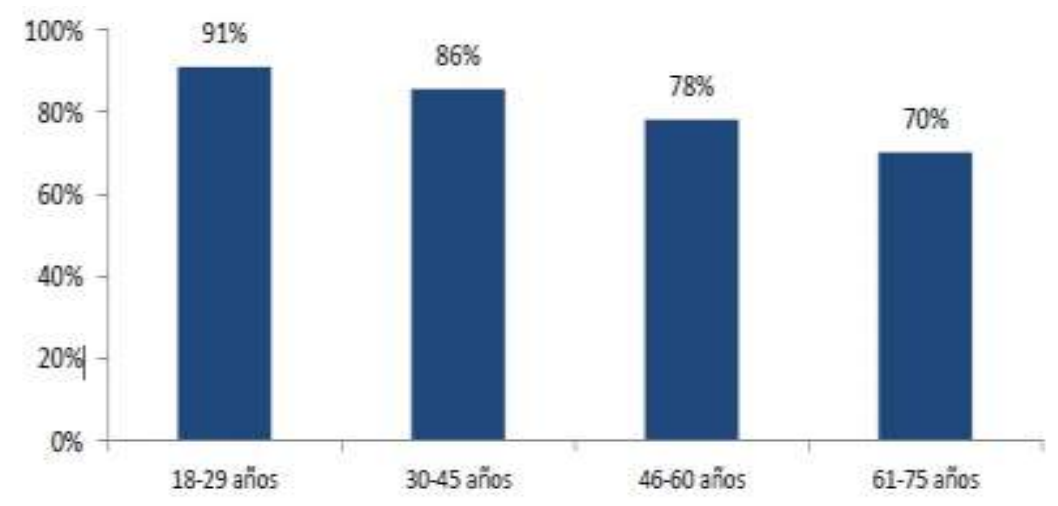

Fig. 1: Distribución porcentual del uso de internet por grupo etario. Fuente: VII Encuesta de Acceso, Usos y Usuarios de Internet Febrero-2016. 
Al observar las tendencias anteriores se optó por incluir la variable género esperando ver el aporte de esta variable a lo observado y en la Figura 3 , se muestra la distribución porcentual de la percepción positiva diferenciada por género para las tres comunas en estudio. Se observa la misma tendencia del género en las tres comunas, indicando que para la percepción positiva no se observa sesgo de género. En la Figura 4, se muestra la distribución porcentual de la percepción negativa y se repite la misma tendencia del género indicando que para la percepción negativa no se observa sesgo de género.
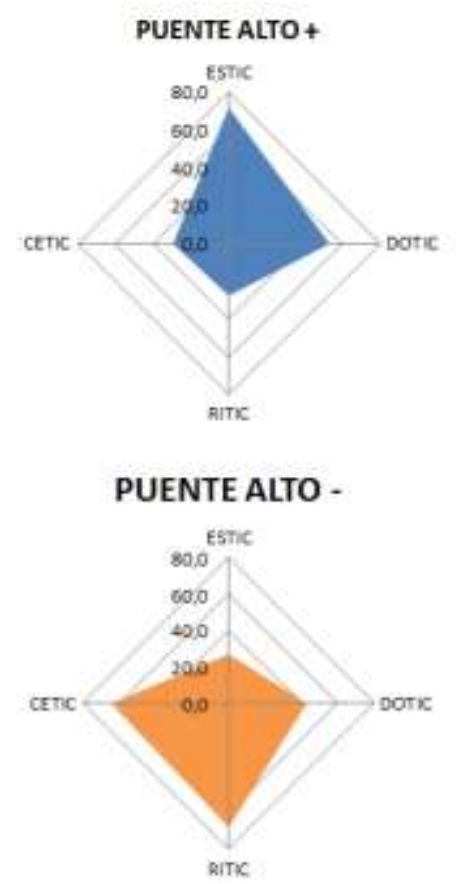

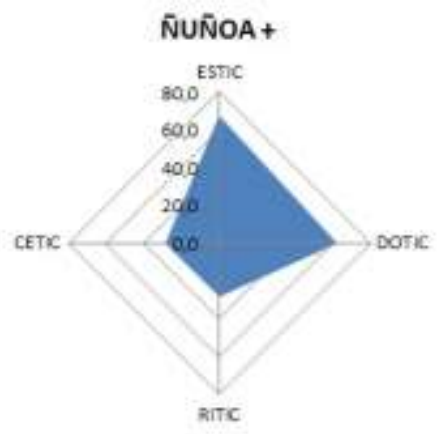

ก̃บก̃OA -

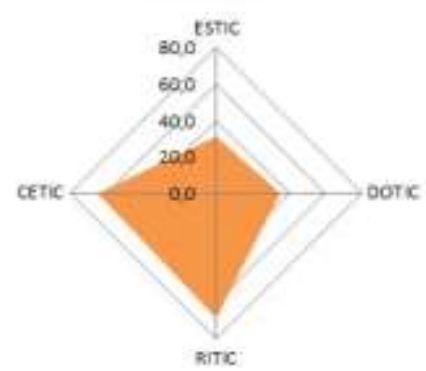

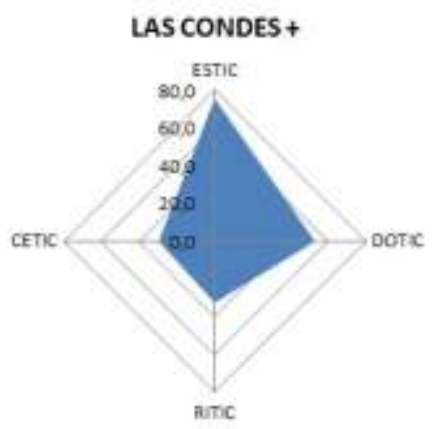

LAS CONDES -

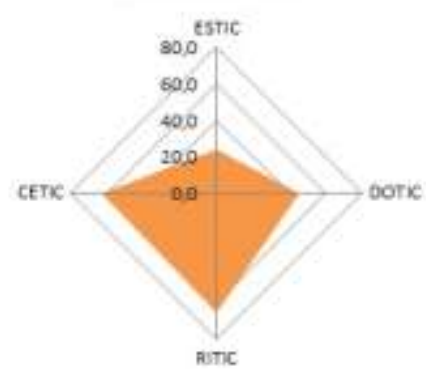

Fig. 2: Distribución radial porcentual de la percepción positiva y negativa por comuna para las cuatro dimensiones medidas.

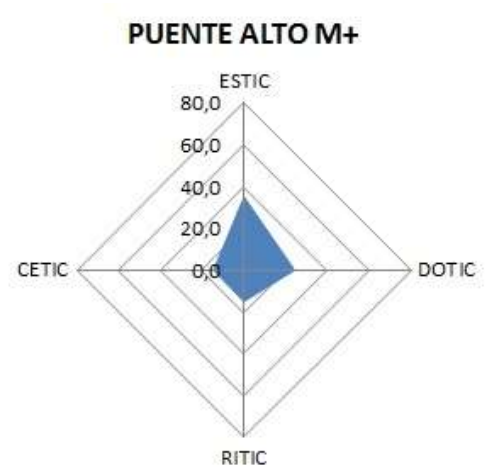

PUENTE ALTO F+

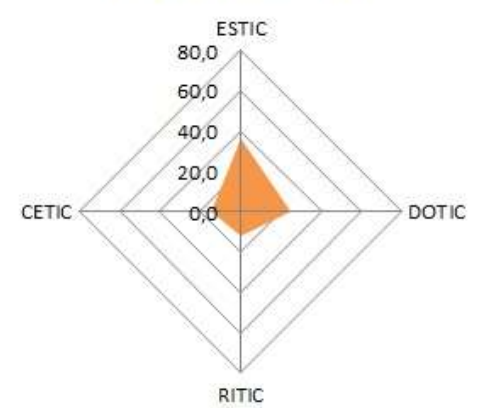

\section{ÑUÑOA M+}

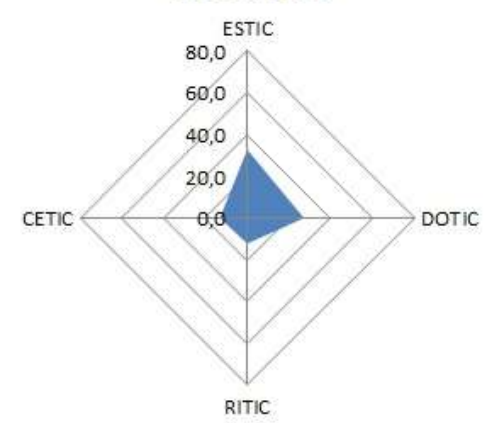

ÑUÑOA F+

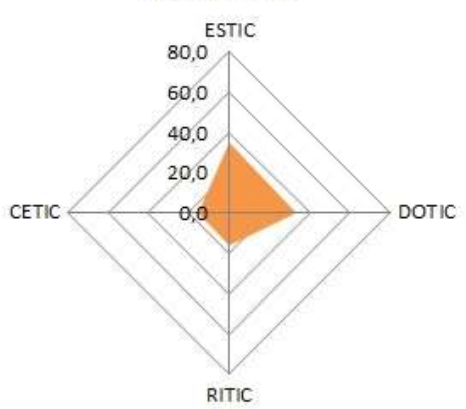

LAS CONDES M+

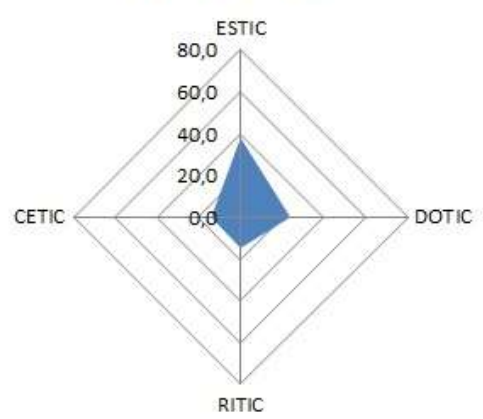

LAS CONDES F+

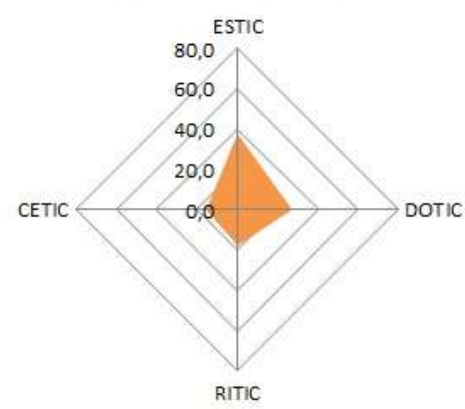

Fig. 3: Distribución radial porcentual de la percepción positiva por género en cada comuna para las cuatro dimensiones medidas. 


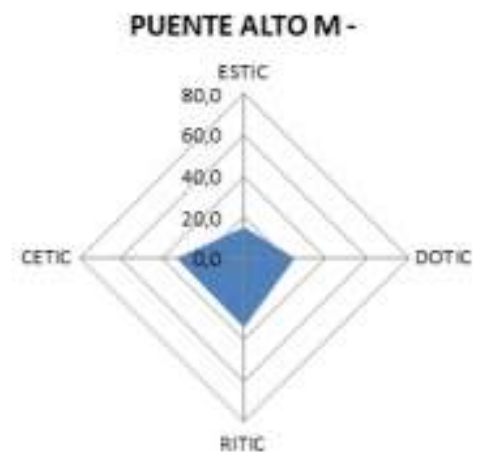

PUENTE ALTO F -

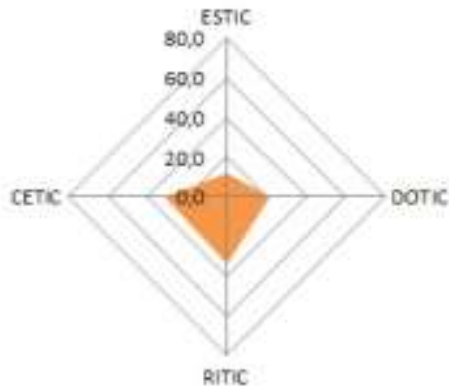

ÑUÑOA M -

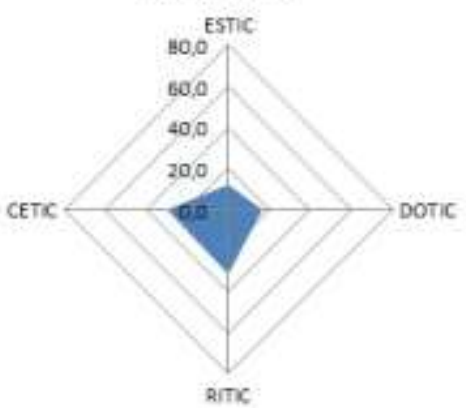

ÑUÑOA F -

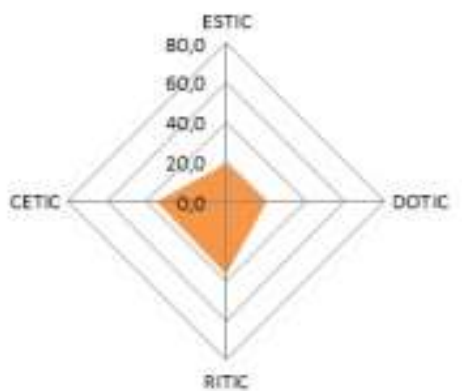

LAS CONDES M-

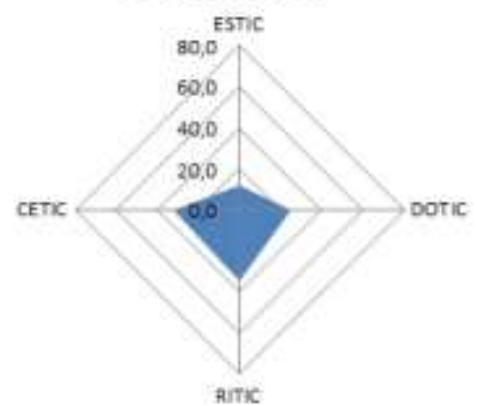

LAS CONDES F -

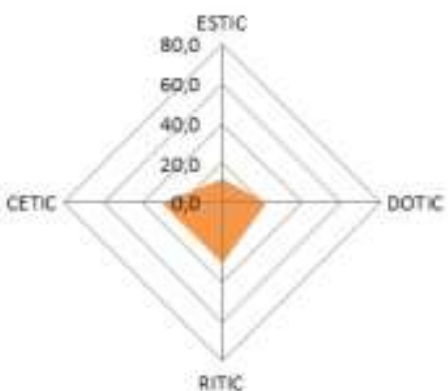

Fig. 4: Distribución radial porcentual de la percepción negativa por género en cada comuna para las cuatro dimensiones medidas.

En la Tabla 4, se puede observar la distribución porcentual por nivel educativo para las tres zonas de estudio, observándose la tendencia natural de a mayor nivel educativo mejor percepción positiva y negativa de las dimensiones. Sin embargo, no hay que dejar de observar que solo en la comuna de Las Condes los indiferentes son bajos o nulos.

Tabla 4: Consolidación de los datos porcentuales por Comuna y Nivel educativo

\begin{tabular}{|c|c|c|c|c|c|c|c|c|c|c|}
\hline & & & Si & & & No & & & lifere & \\
\hline & & $B A$ & $M E$ & $P R$ & $B A$ & $M E$ & $P R$ & $B A$ & $M E$ & $P R$ \\
\hline & Puente Alto & 1,9 & 25,5 & 44,6 & 2,5 & 11,7 & 12,8 & 0,3 & 0,6 & 0,2 \\
\hline ESTIC & Ñuñoa & 0,1 & 21,2 & 46,4 & 0,4 & 9,3 & 21,6 & 0,2 & 0,7 & 0,2 \\
\hline & Las Condes & 0,6 & 15,7 & 59,4 & 0,0 & 5,4 & 18,6 & 0,0 & 0,0 & 0,2 \\
\hline & Puente Alto & 1,4 & 17,7 & 30,4 & 2,7 & 18,5 & 25,4 & 0,5 & 1,6 & 1,7 \\
\hline DOTIC & Ñuñoa & 0,4 & 18,7 & 41,4 & 0,3 & 11,7 & 25,0 & 0,0 & 0,7 & 1,8 \\
\hline & Las Condes & 0,3 & 9,7 & 41,0 & 0,3 & 10,9 & 36,1 & 0,0 & 0,5 & 1,1 \\
\hline & Puente Alto & 1,3 & 9,1 & 17,1 & 2,6 & 27,1 & 38,1 & 0,8 & 1,6 & 2,4 \\
\hline RITIC & Ñuñoa & 0,2 & 10,1 & 18,0 & 0,5 & 20,3 & 47,5 & 0,0 & 0,7 & 2,7 \\
\hline & Las Condes & 0,3 & 6,9 & 25,4 & 0,3 & 13,7 & 51,2 & 0,0 & 0,6 & 1,7 \\
\hline & Puente Alto & 1,8 & 10,5 & 17,2 & 2,6 & 24,9 & 36,9 & 0,2 & 2,4 & 3,5 \\
\hline CETIC & Ñuñoa & 0,2 & 9,3 & 19,0 & 0,1 & 19,7 & 45,1 & 0,4 & 2,1 & 4,0 \\
\hline & Las Condes & 0,2 & 5,0 & 24,5 & 0,5 & 14,8 & 47,7 & 0,0 & 1,4 & 6,0 \\
\hline
\end{tabular}

La Figura 5, muestra los gráficos radiales de las tres zonas de estudio para cada grupo etario, nótese la similitud de la tendencia en las zonas siendo en todos los casos el grupo etario de 30 a 44 años el más representativo y el de 75 o más es menos representativo. Esto indica que es precisamente el grupo de 30 a 44 años que tiene la mayor conciencia del daño que podría tener el uso de la tecnología electromagnética y que no es precisamente falta de información. 

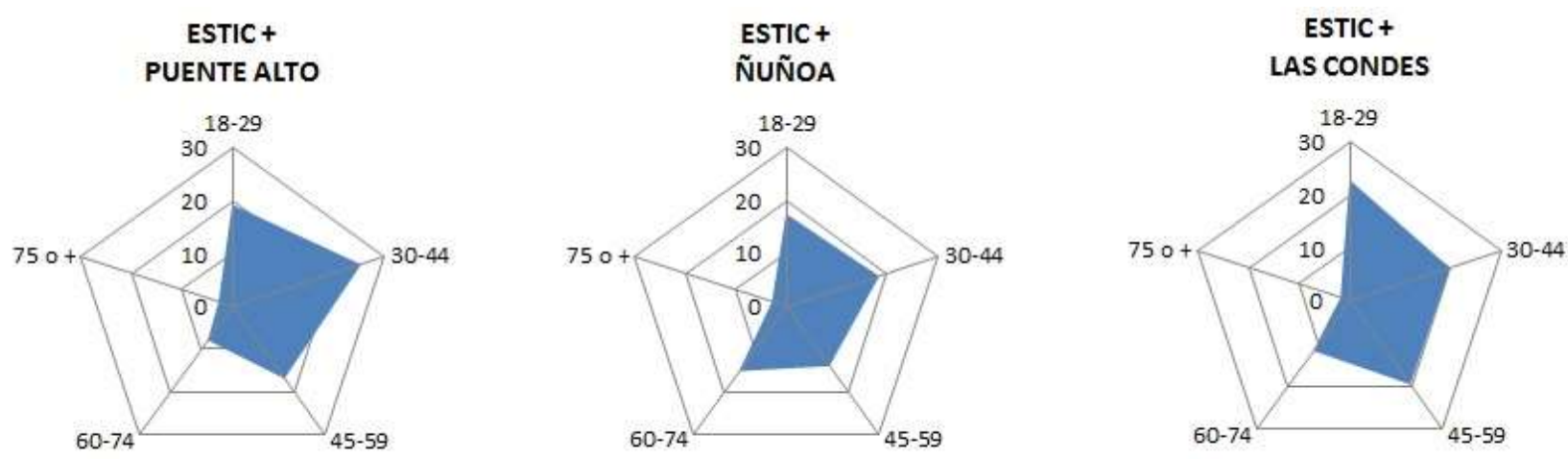

Fig. 5: Resultados porcentuales distribuidos por grupo etario por Comuna para la Dimensión ESTIC

\section{DOTIC + PUENTE ALTO}

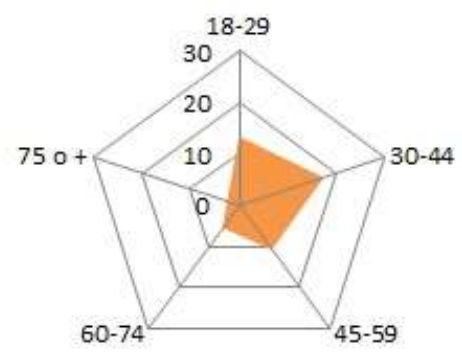

DOTIC + ÑUÑOA

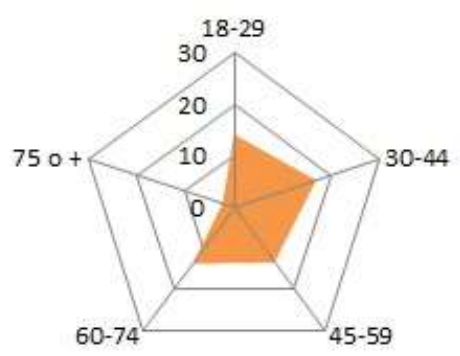

DOTIC + LAS CONDES

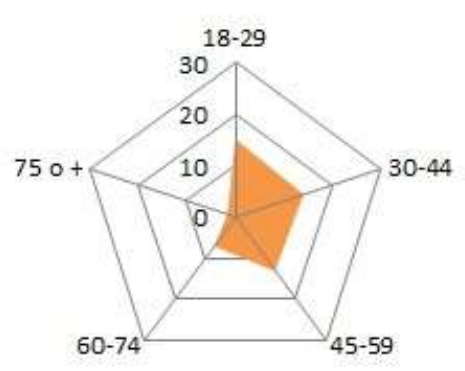

Fig. 6: Resultados porcentuales distribuidos por grupo etario por Comuna para la Dimensión DOTIC

La Figura 6, muestra los gráficos radiales de las tres zonas de estudio para cada grupo etario, nótese la similitud de la tendencia en las zonas, siendo en todos los casos el grupo etario de 30 a 44 años el más representativo y el de 60-74 en menos representativo. Esto indica que existe un conocimiento del principio de funcionamiento de las tecnologías electromagnéticas en el artefacto tecnológico y es precisamente el grupo etaria de 30 a 44 años el que más tiene ese conocimiento y como era de esperarse el grupo con menor conocimiento es el grupo etario de 60 a 74 y el de 75 o más años
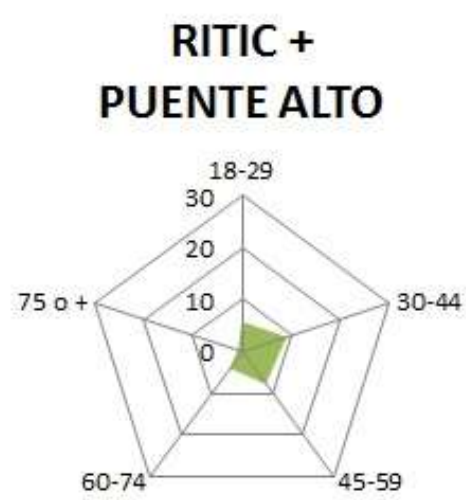

RITIC + ÑUÑOA

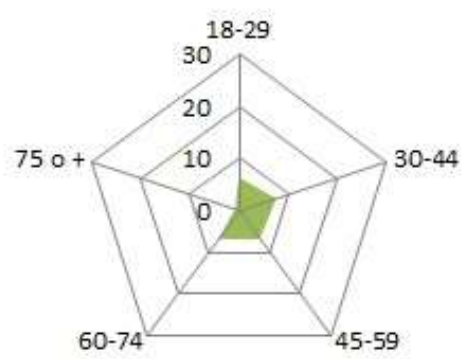

\section{RITIC + LAS CONDES}

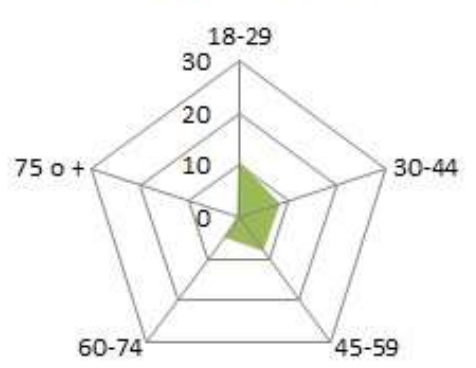

Fig. 7: Resultados porcentuales distribuidos por grupo etario por Comuna para la Dimensión RITIC

En la Figura 7, muestra los gráficos radiales de las tres zonas de estudio para cada grupo etario, donde se observa la dimensión RITIC, nótese la similitud de la tendencia en las zonas, siendo en todos los casos el grupo etario de 30 a 44 años. Es necesario resaltar la percepción como dañina esta tecnología por el grupo etario de 30 a 44 años que es precisamente el grupo que utiliza con mayor conciencia el artefacto tecnológico. En la Figura 8, se muestra los gráficos radiales de las tres zonas para la dimensión CETIC, notándose que el grupo etario de 30 a 44 años es el que más percibe como útil la tecnología electromagnética. 

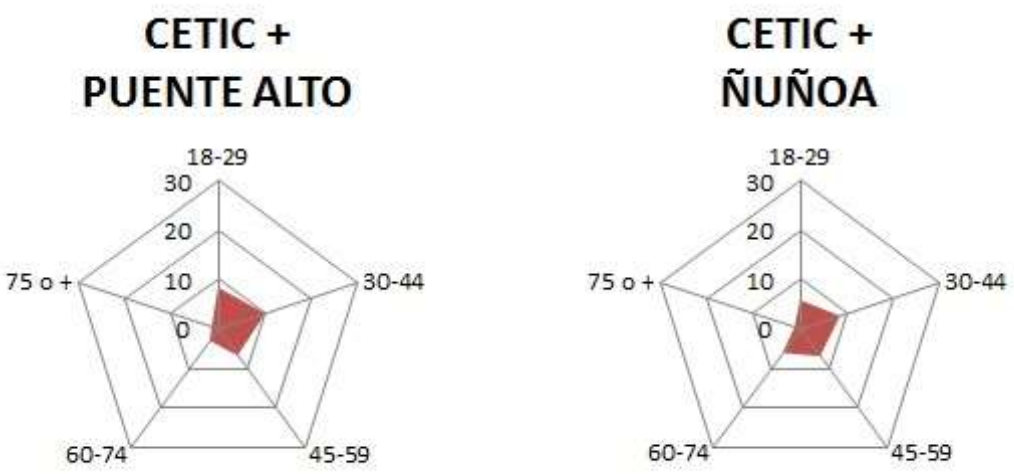

\section{CETIC + LAS CONDES}

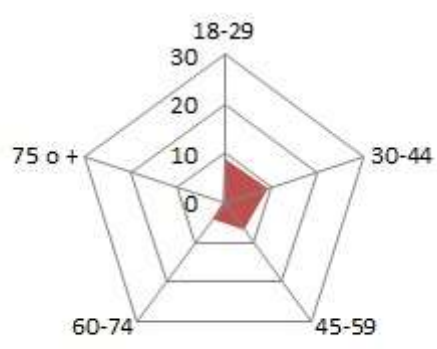

Fig. 8: Resultados porcentuales distribuidos por grupo etario por Comuna para la Dimensión CETIC

\section{CONCLUSIONES}

El estudio muestra la percepción en tres comunas de la región metropolitana para cuatro dimensiones de estudio ESTIC, DOTIC, RITIC y CETIC. El comportamiento de la variable etaria es la esperada y es consistente con lo indicado en el estudio de la subsecretaría de telecomunicaciones, siendo los grupos de mayor percepción positiva de 18 a 29 y de 30 a 44 años, además no se observa sesgo de género por lo que hombres y mujeres tienen la misma percepción en las dimensiones estudiadas.

Por otro lado, la percepción que la tecnología no aporta a las relaciones interpersonales y a la calidad de la enseñanza es notable en las tres zonas de intervención, fortaleciendo la idea que la tecnología no ha logrado colocarse como un elemento robusto en la educación y solo es utilizada como elemento de comunicación o de uso recreativo consistente con el resultado de la encuesta de la súper intendencia de telecomunicaciones, cuando muestra que la primera razón de uso de internet es la comunicación y solo en la tercera posición el uso en la educación. Otro resultado interesante es el alto nivel de polarización alcanzando el $97 \%$ de los que tienen una postura frente al tema tecnología-educación, ese a favor o en contra y solo el $3 \%$ es indiferente.

El estudio transparenta la menor percepción del impacto de la tecnología en la calidad de la enseñanza y en el desarrollo de las relaciones interpersonales, lo que permite al equipo de investigación establecer nuevas intervenciones para definir las razones de dicha percepción, esto hace pensar que se deben redoblar los esfuerzos e innovaciones en las metodologías con intervención de tecnologías de la información que permitan mejorar esta variable.

\section{AGRADECIMIENTOS}

Para la Vicerrectoría de Investigación, Desarrollo e Innovación de la Universidad de Santiago de ChileUSACH (Proyecto DICYT), al Proyecto Basal USA1555 MECESUP-USACH y al Grupo de Investigación en Nuevas Tecnologías (GINT-DTI-USACH) por el importante apoyo al desarrollo de la investigación.

\section{REFERENCIAS}

Araneda-Guirriman, C., Neumann-González, N., Pedraja-Rejas, L. y Rodríguez-Ponce, E., Análisis Exploratorio de las Percepciones sobre los Estilos de Liderazgo de los Directivos Universitarios en el Norte de Chile, Formación Universitaria, doi: 10.4067/S0718-50062016000600013, 9(6), 139-152 (2016)

Bates, A.W., Cómo gestionar el cambio tecnológico. Estrategias para los responsables de centros universitarios, Barcelona, Gedisa (2001)

Cabero, J., Las TIC y Las Universidades: Retos, Posibilidades y Preocupaciones, Revista de la Educación Superior, Universidad de Sevilla, España, 3(135), 77-100 (2005)

Cózar-Gutiérrez, R., De Moya-Martínez, M., Hernández-Bravo, J.A. y Hernández-Bravo, J.R., Conocimiento y Uso de las Tecnologías de la Información y las Comunicaciones (TIC) según el Estilo de Aprendizaje de los Futuros Maestros, Formación Universitaria, doi: 10.4067/S0718-50062016000600010, 9(6), 105-118 (2016)

Lagunes-Dominguez, A., Torres-Gastelú, C. y Flores-García, M., Comparativo del uso de Tecnologías de la Información y Comunicación (TIC) por Profesores de Dos Universidades Públicas de México, Formación Universitaria, doi: 10.4067/S0718-50062015000200003, 8(2), 11-18 (2015) 
López de la Madrid, M.C., Impacto de las tecnologías de la información y la comunicación (TIC) en el Docente Universitario. El caso de la Universidad de Guadalajara, Perspectova Educacional, Formación de Profesores, DOI: 10.4151/07189729-Vol.52-Iss.2 -Art.180, 52(2), 4-34 (2013)

Lopez, M., Sainz, B. y Navazo, M., Nuevas Tecnologáis y su uso en educación, orgs. Linguagem, educação e virtualidade [online]. São Paulo: Editora UNESP; São Paulo: Cultura Acadêmica, 69-91 (2009)

Márquez, P., Impacto De Las Tic En Educación: Funciones Y Limitaciones, 3Ciencias, Departamento de Pedagogía Aplicada, Facultad de Educación Universidad Autónoma de Barcelona (UAB), 2(1), 1-1 (2013)

Mortera, F., Implementación de Recursos Educativos Abiertos (REA) a través del portal TEMOA (Knowledge Hub) del Tecnológico de Monterrey, México, Formación Universitaria, 3(5), 9-20 (2010)

Pinto de la Fuente, M. y González, B., Séptima Encuesta de Acceso, Usos y Usuarios de Internet, Ipsos Public Affairs, The Social Research and Corporate Reputation Specialist, Subsecretaria de Telecomunicaciones (2016)

Polivio, A. y Moreno, G., Uso de las tecnologías de la información en la educación superior, INNOVA Research Journal 2017, 2 (1), 110-123 (2016)

Rodriguez, A., Ramirez, L. y Rodrigues-Martinez-Basile, F., Percepción Social de las Tecnologías Electromagnéticas, Información Tecnológica, http://dx.doi.org/10.4067/S0718-07642016000600023, 27(6), 227-236 (2016)

Rodríguez-Ponce, E. y Pedraja-Rejas, L., Percepciones sobre la Gestión del Conocimiento de Directivos Universitarios de Cuatro Universidades Chilenas, Formación Universitaria, doi: 10.4067/S071850062016000400006, 9(4), 41-52 (2016)

Santana, B., Roddini, C. y De Luca Pretto, N., REA en la educación básica: la colaboración como estrategia de enriquecimiento de los procesos de enseñanza-aprendizaje. Recursos Educacionales Abiertos prácticas colaborativas y políticas publicas, Coordinación Editorial: Maracá - Educação e Tecnologias | Casa de la Cultura Digital, 119-127 (2013)

Silva-Peña, I., Borrero, A.M., Marchant, P., González, G. y Novoa, D., Percepciones De Jovenes Acerca Del Uso de Las Tecnologias de Informacion en El Ambito Escolar, Revista Ultima Década, Centro de Estudios Sociales CIDPA, http://dx.doi.org/10.4067/S0718-22362006000100003, 14(24), 37-60 (2006)

Starobinas, L., REA en la educación básica: la colaboración como estrategia de enriquecimiento de los procesos de enseñanza-aprendizaje. Recursos Educacionales Abiertos prácticas colaborativas y políticas publicas, Coordinación Editorial: Maracá - Educação e Tecnologias | Casa de la Cultura Digital, 119-127 (2013)

Tapasco, O. y Giraldo, J., Estudio Comparativo sobre Percepción y uso de las TIC entre Profesores de Universidades Públicas y Privadas, Formación Universitaria, doi: 10.4067/S0718-50062017000200002, 10(2), 3-12 (2017) 
